\title{
De-biasing interferometric visibilities in VLTI-AMBER data of low SNR observations ${ }^{\star}$
}

\author{
G. Li Causi ${ }^{1}, \mathrm{~S}$. Antoniucci ${ }^{1,2}$, and E. Tatulli ${ }^{3,4}$ \\ 1 INAF - Osservatorio Astronomico di Roma, via Frascati 33, 00040 Monteporzio Catone, Italy \\ e-mail: licausi@oa-roma.inaf.it \\ 2 Università degli Studi di Roma "Tor Vergata", via della Ricerca Scientifica 1, 00133 Roma, Italy \\ 3 INAF - Osservatorio Astrofisico di Arcetri, Largo E. Fermi 5, 50125 Firenze, Italy \\ ${ }^{4}$ Laboratoire d'Astrophysique Observatoire de Grenoble, BP 53, 38041 Grenoble Cedex 9, France
}

Received 11 April 2007 / Accepted 26 November 2007

\section{ABSTRACT}

\begin{abstract}
Aims. We have found that the interferometric visibilities of VLTI-AMBER observations, extracted via the standard reduction package, are significantly biased when faint targets are concerned. The visibility biases derive from a time variable fringing effect (correlated noise) appearing on the detector.

Methods. We have developed a method to correct this bias that consists in a subtraction of the extra power caused by such correlated noise, so that the real power spectrum at the spatial frequencies of the fringing artifact can be restored.

Results. This pre-processing procedure is implemented in a software program called AMDC, available to the community, which is to be run before the standard reduction package. Results obtained on simulated and real observations are presented and discussed.
\end{abstract}

Key words. instrumentation: interferometers - instrumentation: detectors - methods: data analysis - techniques: interferometric

\section{Introduction}

Astronomical interferometry consists in combining in phase the light from two or more telescopes, to produce an interferogram at the focal plane, i.e. an image composed of several fringes, which is recorded by the detector. The presence of a common electronic readout effect known as fringing, normally negligible in single telescope observations, is very dangerous for such interferometric data.

The term fringing is commonly used to indicate a spurious pattern composed of parallel fringes overlaid on the image. It can be stable or variable with time, both in phase and amplitude, is sometimes variable in period and orientation, or it can even be non-periodic, so that it is configurable as a structured random noise, also called correlated noise ${ }^{1}$.

Considering such fringing instead of the true interferometric signal may lead to incorrect visibility estimates, especially when dealing with low SNR (signal to noise ratio) observations.

We have discovered the presence of this correlated noise effect in many observations of the interferometric instrument VLTI/AMBER (Petrov et al. 2007), especially those characterized by a very low SNR; in these data, the main fringing component happens to have the same period as the true interferogram, thus biasing the visibility extracted by the standard AMBER processing package amdlib.

\footnotetext{
* Based on VLTI-AMBER observations.

1 In astronomical imaging there is another effect known as fringing, where irregular fringes appear on the sky background. This is caused by the light of the sky emission lines that interferes with the faces of the detector window. We do not refer to this particular fringing effect in this work.
}

The design of AMBER and its data reduction software were actually conceived taking into account the presence of the FINITO fringe tracker (Gai et al. 2004), i.e. with the assumption of stable fringes ensuring long integration times and photon-noise regimes. Because of problems related to structural vibrations within the VLTI, FINITO is not yet available for observations with the unitary telescopes (UTs), and this explains why, even for faint sources, AMBER is currently used with short integration times that often force the instrument to work at a fringe SNR level close to one. In such a situation, the scientifically useful signal is basically comparable to the fringing, so that the correlated noise can no longer be neglected in the data processing.

We present here a method that we have developed to remove the bias introduced to the VLTI-AMBER visiblities by the correlated noise.

After a short presentation of the AMBER instrument (Sect. 2), we present a brief report of the standard data reduction procedures (Sect. 3); then, we describe the fringing artifact (Sect. 4) and the method we have developed to remove it (Sect. 5). Finally, we discuss the results obtained applying the correction to real and simulated data (Sect. 6).

\section{The AMBER instrument}

AMBER, the first-generation near-infrared interferometric instrument in operation at the ESO Very Large Telescope Interferometer (VLTI), has a beam combiner fed by three fibers, capable of simultaneously providing multi-wavelength visibilities and closure phases (Petrov et al. 2007). AMBER works by combining the three beams on the same area of an IR array, thus forming three superimposed fringe patterns of known spatial periods (Tatulli et al. 2007). This approach, called multiaxial 


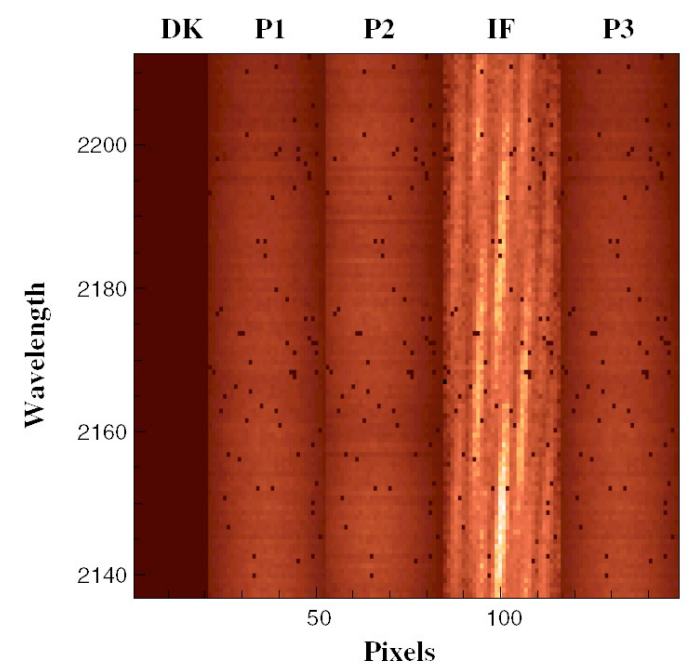

Fig. 1. The five channels of an AMBER image: from left to right the darkened channel DK, which is a physically masked region of the detector, the photometries P1 and P2, the interferogram IF, the photometry P3 (adapted from Tatulli et al. 2007).

combination allows the information of the photometry of the three beams, and the three correlated fluxes to be encoded in a small detector area. Spectral dispersion is added along the orthogonal direction, with a spectral window that depends on the resolution, selected among $R=30$ (LR-mode), $R=1500$ (MRmode) and $R=12000$ (HR-mode). This results in frames that are divided in five vertical regions or "channels", as shown in Fig. 1, where the OPD (optical path difference) is along the $x$ axis and wavelengths along the $y$-axis. Three channels carry the photometric information, one for each telescope, while the interferometric channel contains the overlay of the three interferograms, related to the three baselines; these are characterized by different fringe periods, orientations and shapes, which are extracted by a custom data reduction algorithm (see Sect. 3).

Due to the complex optical train, to the spectral dispersion and mainly to the OPD instabilities caused by the atmospheric piston variations (i.e. the zero-order phase variations over different telescopes), AMBER is limited to a $K$-band magnitude ranging from 4.5 to 5.5 , in MR-mode with the UTs, depending on seeing conditions and adaptive optics performance, while the single frame integration time (DIT) is restricted to a range from 25 to $100 \mathrm{~ms}$; the fringe-tracker FINITO (Gai et al. 2004) is foreseen in the near future to overcome these limitations ${ }^{2}$.

\section{Observations and data reduction with AMBER}

We have analyzed several AMBER observations (in particular, observations taken in medium spectral resolution mode, where the number of photons per spectral channel is drastically reduced) and all of them display some correlated noise fringing. In order to illustrate the correlated noise problem and the correction procedure, we will refer here to the observations of the young stellar object Z CMa A (Koresko et al. 1991), which are characterized by a very low fringe SNR and by a strong noise artifact. The target (a source with a slightly variable $K$ band magnitude around $4.2 \mathrm{mag}$ ) has been observed using the VLTI UT1-UT2UT4 baselines in MR-mode $(R=1500)$, with a spectral window centered at $2.16 \mu \mathrm{m}$ and using a DIT of $50 \mathrm{~ms}$.

${ }^{2}$ Note that FINITO is already available with the auxiliary telescopes.
A part of the frame sequence from the observations is reported in Fig. 2: panel $a$ displays measurements on the calibrator $($ HR2379, diameter $=1.75$ mas, $K$-band magnitude $=2.24)$, while panel $b$ shows frames acquired on the target $\mathrm{Z} \mathrm{CMa} \mathrm{A.} \mathrm{We}$ can note by direct visual inspection that interferometric fringes appear on the calibrator frames, while no fringes seem to be present on the target observations.

In order to derive the interferometric visibilities we used the standard AMBER reduction pipeline amdlib, described in detail by Tatulli et al. (2007). The procedure used in the pipeline is based on the so-called "P2VM matrix" method (Millour et al. 2004), in which the three baseline fringe patterns are disentangled by solving a linear system describing the fringe packets for each spectral channel separately.

Following the standard data reduction procedure, we obtain calibrated visibilities of $1.26 \pm 0.08,0.88 \pm 0.04$ and $0.83 \pm 0.04$ for the UT1-2, UT2-4 and UT1-4 baselines, respectively. These non-zero values appear to be in contrast with the apparent lack of fringes in the frame sequence shown in Fig. 2 panel b, but using a proper visual enhancement (see footnote 3 ) at least two fringe systems can be detected in the target observations (Fig. 2, panel c). However, the visibility measured on the first baseline is greater than one, while by definition the normalized visibility should be between 0 and 1 . Two main causes could explain this inconsistent result: $(i)$ a change of atmospheric conditions between the observation of the science target and the calibrator, which prevents us from properly correcting the visibility for the atmospheric transfer function, or (ii) the presence of detector artifacts that bias the visibility estimate. In the next section, we show that this latter effect is indeed present in the Z CMa A data and that this situation is commonly found in all AMBER low SNR observations.

\section{The detector artifact}

To investigate the cause of the problematic results described above, we performed a 2D Fourier analysis of the frames. Looking at the averaged power spectrum (PS) of the interference channels for the calibrator and the target (Fig. 3, panel a) we easily recognize the three baseline peaks plus the continuum peak at zero frequency, but we also notice some extra power peaks in other locations, as indicated in the figure. Remarkably, we find exactly the same extra peaks both in the photometric channels and in all the channels of the dark observations, where no fringes are expected (last three panels in Fig. 3). Even the PS of the physically masked channel DK shows the same peaks, which means that they are not due to light interference but are spurious artifacts coming from the detector electronics or readout process. The most relevant issue about these artifacts is that the brightest peaks happen to lie at the same spatial frequencies as baseline 1 and, in part, as baseline 3 . Thus, even in the absence of light on the detector, there are some artificial "detector fringe patterns" in the AMBER frames, which have the same spatial periods as the baseline fringes.

The amdlib software cannot distinguish this fringing from the true baseline interferograms, because its analysis is based on line-by-line fringe fitting, allowing for random fringe orientation as induced by the atmospheric piston variation. This is the reason why we obtain incorrect visibilities from the amdlib extraction: the algorithm fits the fringing artifact instead of the true signal because this latter, in the case of low SNR targets, has equal or smaller amplitude than the former.

We point out that this pattern is not removed by the normal dark subtraction operated by amdlib because it is rapidly variable 


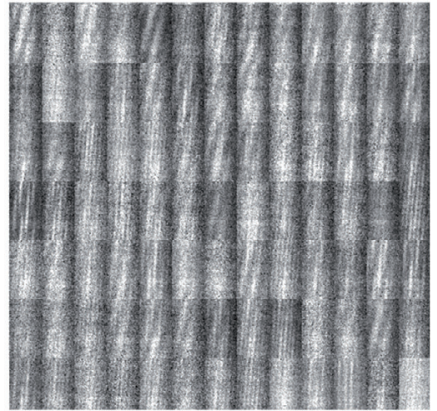

Calib (HR2379_B02)

a)

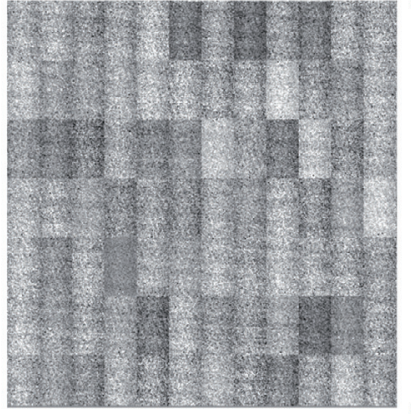

Target (ZCMa A)

b)

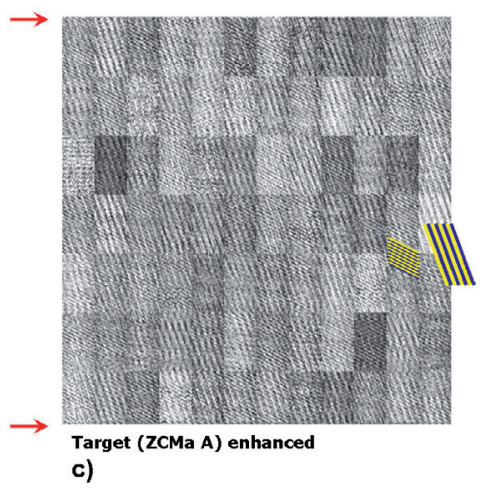

c)

Fig. 2. Time sequence of the interferometric channel for the calibrator HR2379 a) and for the target Z CMa A b). Panel c) shows an enhancement of the same sequence for the target (see footnote 3): at least two fringe systems are visible, as indicated.

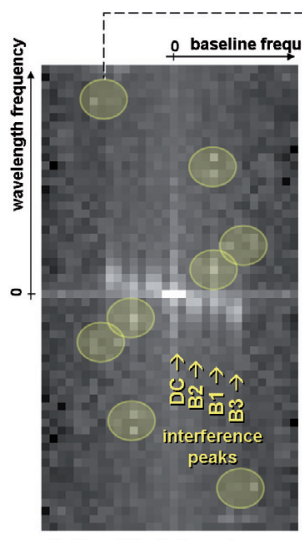

a)

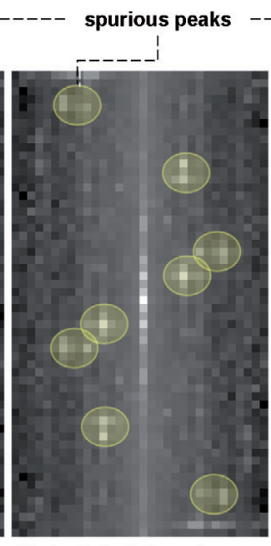

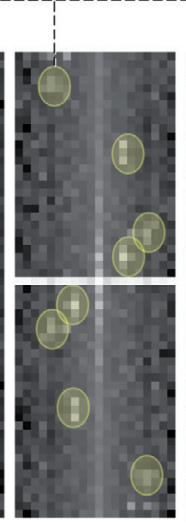

Dark - DK channel

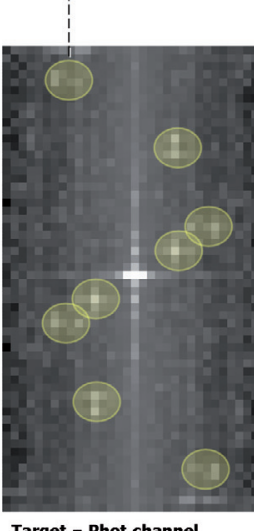

d)

Fig. 3. Panel a): comparison between the average power spectra of the interference channel from the observations of the calibrator and of the target. The origin of the frequency plane is at the center, with baseline frequencies along the $x$-axis and wavelength frequencies along the $y$-axis. The three baseline peaks are visible in the calibrator (B2 - B1 - B3 labels), while only the baseline 1 signal is clearly visible in the target. Some spurious power peaks can be identified spread over the plane (circles), which appear at the same positions for both the calibrator and target. Noticeably, they also appear in the dark observations (panel b)), in the masked channel DK (panel c)) and in the photometric channels (panel d)), where no fringes are expected.

in time and thus has a different phase shift in each frame. Consequently, this spurious pattern is not present in the average dark frame, and the dark subtraction is not able to remove it.

Figure 4 shows a direct comparison between the frames of the target and the frames of a photometric channel of the dark observation (where we would expect no fringes): we clearly see exactly the same fringe systems, thus confirming that our Z CMa A observations are dominated by the correlated noise and that all the fringes displayed in Fig. 2, panel c, are spurious.

\section{Fringing removal: the AMDC software}

The fringing affecting AMBER data is likely to be caused by some electromagnetic interferences (EMI) corrupting the detector read-out process (see e.g. Mardones 2007; and Beckmann 2007). Hereafter, we present our approach to correcting this problem via software.

The procedure we have developed is implemented in the AMDC tool, which stands for "AMBER detector cleaner". AMDC effectively removes the fringing artifact from AMBER raw frames, thus allowing users to properly reduce observations obtained in poor instrumental conditions.
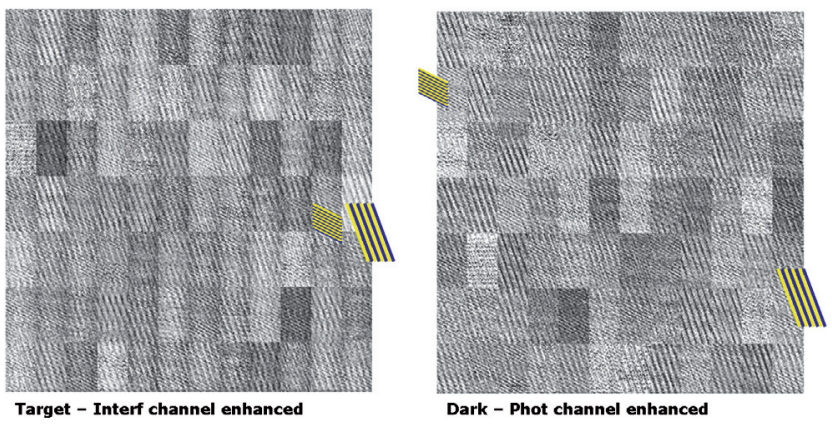

Fig. 4. Comparison between the fringe systems observed in the interference channel of the target and the ones observed in a photometric channel of the dark observations (enhanced, see footnote 3): both of them are artifacts of the AMBER detector.

The program is written in IDL (Interactive Data Language) and is downloadable at http://www.mporzio.astro.it/ $\sim$ licausi/AMDC/.

AMDC works as a pre-processing tool to be run on the original AMBER frames before the standard amdlib reduction is performed. It creates a copy of the original data set where the fringing is no longer present, so that the standard processing can be performed on these corrected data. 
Our approach consists in $(i)$ marking the locations of the artifacts in the average PS, then (ii) replacing a modelled PS estimate within the marked frequency regions on a frame-by-frame basis and finally (iii) rebuilding the image frames via inverse Fourier transform.

Working on power spectra, this method will effectively subtract the contribution to the power caused by the correlated noise in all the involved frequencies, but cannot restore their original phases, for the reasons we will explain below. Nonetheless, the reconstruction error of both the modulus and phase of the final visibility is negligible providing that the artifact peaks do not overlap with the baseline peaks in the PS. This situation that can be easily avoided by setting a suitable non-zero OPD during the fringe centering stage at the beginning of the AMBER observational procedures (in fact, a variation of the central OPD causes the science peaks to shift vertically in the frequency plane, thus placing them away from the artifact regions). In contrast, in the unlucky case where artifact and interferometric peaks do overlap in the PS, the algorithm will still allow amdlib to restore the correct average $V^{2}$, but not the single visibilities $V_{i}$ of each frame.

From a mathematical point of view, we proceed as follows. At first we build a binary mask to mark the frequencies affected by the artifacts, which are identified on the average PS of the dark frames by an algorithm that repeats a sigma-clipping three times followed by a morphological dilation operator, so as to mark both the main peaks and their tails.

For each photometric channel of the dark observations $\left(D_{p, i}\right)$, we compute the average (over the $n$ frames) power $\widehat{D}_{p}$ :

$\widehat{D}_{p}=\frac{\sum_{i}^{n}\left|F T\left(D_{p, i}\right)\right|^{2}}{n}$.

Then we derive the binary mask $M_{p}$ marking the frequencies where the power differs from the median by more than a threshold of $K$ standard deviations. The median is computed on a column-by-column basis, given the vertical symmetry of the PS.

$M_{p}\left(v_{x},\left\{v_{y}:{\widehat{D^{\prime}}}_{p}\left(v_{x}, v_{y}\right)>\left[K * \sigma_{\widehat{D}_{p}^{\prime}\left(v_{x}, *\right)}\right]\right\}\right)=1,0$ elsewhere

where

$\widehat{D}_{p}^{\prime}\left(v_{x}, *\right)=\widehat{D}_{p}\left(v_{x}, *\right)-\operatorname{MEDIAN}\left(\widehat{D}_{p}\left(v_{x}, *\right)\right)$

and $\sigma$ is a robust standard deviation estimator. Using this procedure we mark the power peaks, but we also need to mark their tails. Thus, we apply Eq. (2) again, using a lower threshold $K \sigma$, but only in the pixels surrounding the spurious peaks we have previously identified, then iterate this procedure if needed.

Finally, the masks $M_{p}$ computed for each photometric channel are median-averaged to build a single mask $\bar{M}$ that we will use for all the channels.

The above procedure is repeated for each type of AMBER observation file (calibrator, target, P2VM calibration, spectral calibration) in order to produce the right mask to be used for each of them (Fig. 5).

Analyzing the PS of the single frames we also notice that the detector pattern is characterized by a highly variable amplitude, so that some frames appear heavily affected by artifact, while others do not show any bias. This means that we cannot simply subtract the average PS of the dark frames from the PS of each target frame; instead we need an estimate of the instantaneous artifact PS. To get this estimate we assume that the spurious power in the interferometric channel of a frame is given by the average of the spurious power in the photometric channels of the same frame. We obtain this latter as the difference between
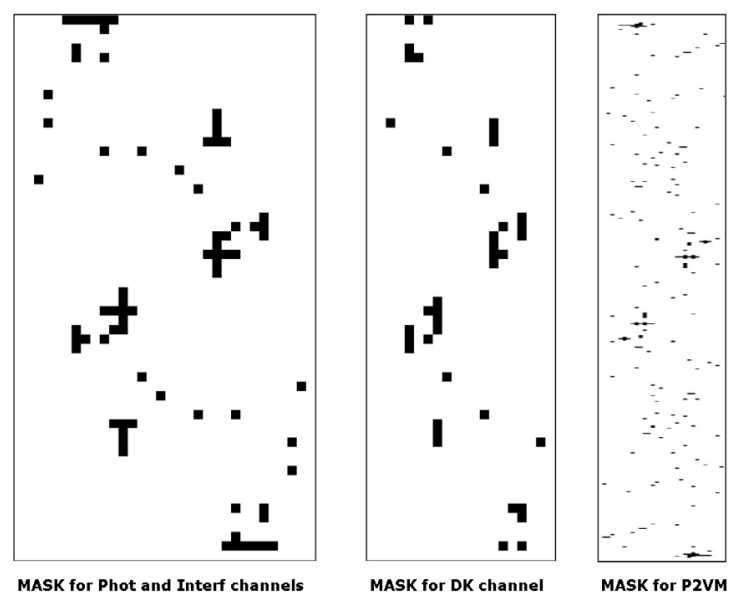

Fig. 5. Examples of masks, computed from the average power spectrum of the dark frames, used to remove the detector pattern artifact from the interferometric and photometric channels (left), from the masked channel DK (middle) and from the P2VM frames (right).

the currently measured PS and a model of the uncorrupted PS, $W_{p}$. This model is built from the average PS of the P2VM files where only one photometric channel at a time is lit (Tatulli et al. 2007). Indeed, in these files the other channels are darkened, so that their PS only contain the simultaneous spurious peaks, and we subtract the average of these latter from the PS of the opened channel. This way we get the three perfectly cleaned photometric PS to be used as $W_{p}$ models.

In formulae, since these models and the observed PS of the photometric channels of the target $\widehat{T}_{p, i}$ differ for a global constant and a scaling factor, we adapt the model to the measured PS by a linear fit, restricted to the uncorrupted frequencies, i.e. where $\bar{M}\left(v_{x}, v_{y}\right)=0$ :

$\left|\left[a_{0} * W_{p}+a_{1}\right]-\widehat{T}_{p, i}\right|^{2}<\varepsilon$

where $a_{j}$ are the coefficients of the fit.

Thus, the estimates of the instantaneous artifact power $A_{p, i}$ on the masked frequencies (where $\bar{M}=1$ ) are given by:

$A_{p, i}=\widehat{T}_{p, i}-\bar{W}_{p}$

and zero elsewhere, where $\bar{W}_{p}$ are the best fit models.

Finally, we derive a cleaned PS of each frame by directly subtracting the $A_{p, i}$ from the photometric channels $p$ and their median-average $\bar{A}_{p, I}$ from the interferometric channel $I$ :

$\widehat{T}_{p, i}^{\text {Corr }}=\widehat{T}_{p, i}-A_{p, i}$

$\widehat{T}_{I, i}^{\text {Corr }}=\widehat{T}_{I, i}-\bar{A}_{p, i}$.

In summary, we are subtracting from each frame the power of the detector noise present at the moment of the frame readout.

Figure 6 shows the result of this process applied to our data: we note that $(i)$ the only remaining peaks in the PS of the calibrator are the three baseline signals, (ii) no spurious peaks remain in the PS of the corrected dark frames and (iii) in the target PS we clearly see the clean peaks of the first baseline, which carries the dominant interferometric signal of these data (zoomed panel in the figure).

A thorough analysis has shown that it is not possible to recover the uncorrupted phase information $\varphi_{I, i}\left(v_{x}, v_{y}\right)$, unless each pixel is tagged with a precise read-time stamp, which is not available in the AMBER files. Indeed, the external 


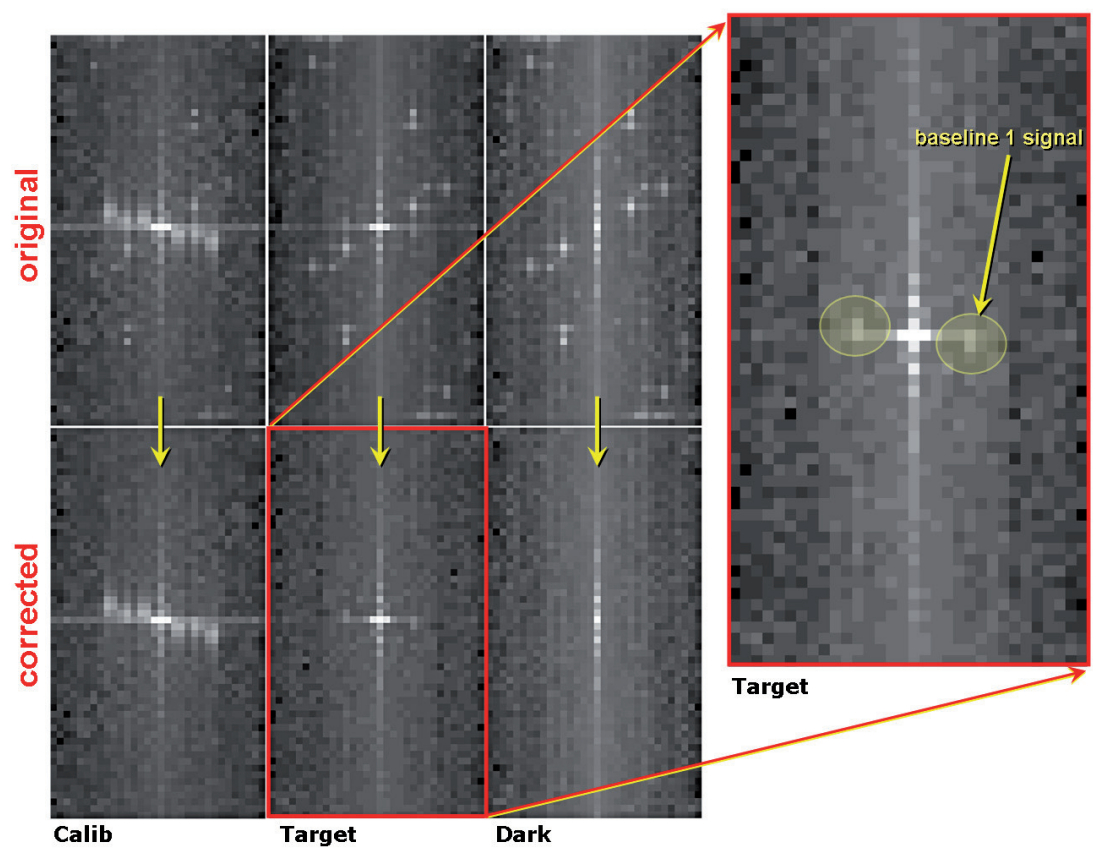

Fig. 6. Removal of the artifact peaks from the power spectra of calibrator (left), target (middle) and dark frames (right): original power images are displayed on the top row and corrected ones at bottom. The faint interferometric signal in baseline 1 of our target is now clearly visible (zoomed panel).

EMI $E(t)$ combines with the complex pixels readout temporal sequence $U(t)$ to produce a different spatial modulation $V(x, y)$ for each frame readout. In the case of AMBER, the detector is read by a double correlated sampling, which follows a "Reset - Non Destructive Read - Destructive Read" scheme, where the two readout sequences have different timings and only the difference between them within the channel windows is transferred. This implies that the final fringing pattern has a spectrum of spatial frequencies with a phase distribution that is arbitrarily complex, even if the EMI is monochromatic. This prevents us from recovering the phased frequency spectra at the artifact frequencies, thus affecting the single frame visibilities in case of coincidence with baselines frequencies. This is the reason why a suitable OPD correction must be adopted, as already noted, to avoid any possible overlapping of baseline and artifact signals in the PS.

At the end of the process the reconstructed image frames, which no longer contain the detector artifact, are computed by means of an inverse Fourier Transform and put in place of the original (corrupted) frames in the AMBER file:

$T_{I, i}^{\mathrm{Corr}}=F T^{-1}\left(\left[\widehat{T}_{I, i}^{\mathrm{Corr}}, \varphi_{I, i}\right]\right)$

$T_{p, i}^{\text {Corr }}=F T^{-1}\left(\left[\widehat{T}_{p, i}^{\text {Corr }}, \varphi_{p, i}\right]\right)$,

where the phases $\varphi_{*, i}\left(v_{x}, v_{y}\right)$ are the original ones.

Figure 7 shows the result of the AMDC correction on the frame sequence of $\mathrm{Z} \mathrm{CMa} \mathrm{A}$ : we clearly $\mathrm{se}^{3}$ that the spurious fringes are removed and the real interferometric fringes appear, while

\footnotetext{
3 Note: for this figure, as for some previous figures displaying frame sequences, we have used the AMDC enhancement function because of the very low contrast of the fringes, hardly visible even on the screen; this is obtained by magnifying, by one order of magnitude, the power at the frequencies identified by the mask, or that of the baselines peaks. Note as well that a "visual-inspection" of the frames is in general suitable for a quick qualitative analysis of the data, since fringes might be present without being strong enough to be seen above the noise level on the screen, as observed here for the second and third baselines of Z CMa A.
}
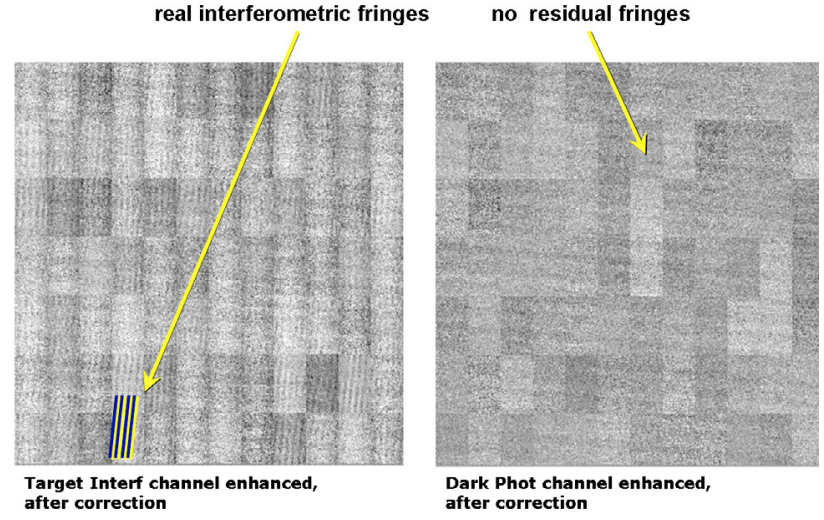

Fig. 7. AMDC enhancement of the frame sequence after removal of the detector artifact: the true interferometric fringes now appear on the target (left), while no residual fringes remain on the dark frames (right). Compare them to Fig. 4.

no residual fringes are visible in the sequence of dark frames, thus confirming that what we obtain is not an artifact of our processing.

The correction procedure described for the target frames must then be applied to all the frames of the data set, including the spectral calibration files, the P2VM calibration files, the calibrator files and the dark observations themselves. Such operations have to be done at a pre-processing stage, before launching the standard amdlib sequence (Altariba et al. 2006), consisting in spectral offset computations, building of the P2VM matrix and visibility extraction.

This procedure is performed in a semi-automatic fashion by AMDC, which also provides some functions for a first analysis of the data, aimed at finding out whether any correlated noise is

For a thorough data analysis, a quantitative parameter, namely the SNR fringe contrast as defined in Eq. (20) of Tatulli et al. (2007), is the criterium to be used. 
Table 1. Comparison of corrected and uncorrected visibilities. Raw and calibrated visibilities, averaged over wavelengths, before and after artifact correction by AMDC, resulting from amdlib extraction with $50 \%$ selection by flux criterium, then $50 \%$ selection by SNR, then all frames binning.

\begin{tabular}{cccccccc}
\hline \hline & Baselines & \multicolumn{3}{c}{ Raw visibilities } & \multicolumn{2}{c}{ Calibrated visibilities } \\
\hline & $u, v$ & \multicolumn{2}{c}{ Z CMa A } & \multicolumn{2}{c}{ HD2379 } & \multicolumn{2}{c}{ Z CMa A } \\
$\#$ & (meters) & Uncorrected & Corrected & Uncorrected & Corrected & Uncorrected & Corrected \\
\hline 1 & $24.8,49.3$ & $0.68 \pm 0.04$ & $0.45 \pm 0.01$ & $0.51 \pm 0.01$ & $0.46 \pm 0.01$ & $1.26 \pm 0.08$ & $0.81 \pm 0.03$ \\
2 & $88.4,13.1$ & $0.60 \pm 0.01$ & $0.62 \pm 0.01$ & $0.60 \pm 0.03$ & $0.60 \pm 0.03$ & $0.88 \pm 0.04$ & $0.94 \pm 0.02$ \\
3 & $113.2,62.4$ & $0.58 \pm 0.02$ & $0.58 \pm 0.02$ & $0.52 \pm 0.02$ & $0.52 \pm 0.02$ & $0.83 \pm 0.04$ & $0.67 \pm 0.02$ \\
\hline
\end{tabular}

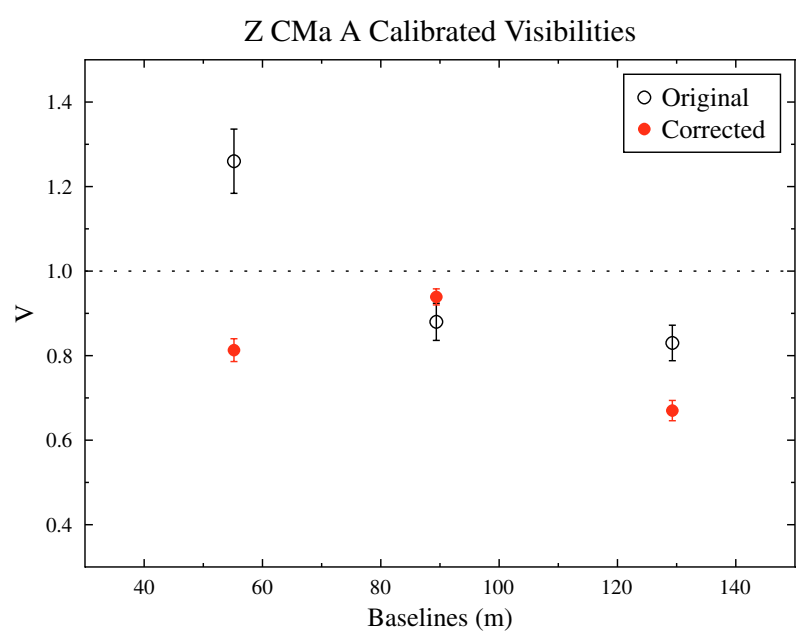

Fig. 8. Visibilities of Z CMa A before and after the AMDC correction; noticeably, no baselines have visibilities greater than one after the correction.

present and improving visualization of this noise. Procedures are available for displaying the frames sequence and their average PS, for automatically or manually defining the binary mask, for applying the artifact correction, for creating the restored FITS files, and for enhancing the visualization of the fringing.

\section{Corrected visibilities}

The application of the AMDC correction to Z CMa A data provides the results reported in Table 1, which shows the raw visibilities of target and calibrator before and after the artifact removal.

The target and the calibrator are not equally affected, as expected for sources with different SNR: in particular, we find a relative bias up to $50 \%$ for the uncorrected raw visibilities of $\mathrm{Z} \mathrm{Cma} \mathrm{A}$, while the results on the calibrator appear to be only slightly corrupted. This explains the overestimate of the calibrated visibilities of $\mathrm{Z} \mathrm{CMa} \mathrm{A}$ that we reported in Sect. 3; the values we obtain after the fringing removal are $0.81 \pm 0.03$, $0.94 \pm 0.02$ and $0.67 \pm 0.02$, without meaningless values greater than one on the first baseline (Fig. 8).

Moreover, our analysis has shown that dispersion of the visibility among the observation files is noticeably reduced after the correction; this also partly explains the large visibility dispersion normally found in AMBER observations of weak sources, which is therefore not due to just the VLTI instrumental/atmospheric OPD variations. Unfortunately, we have not been able to evaluate the AMDC results on the closure phase, given the extremely low SNR of the data and the requirement that fringes must be present in the three baselines at the same time.

The same correction procedure has been applied to other AMBER observations of low flux targets (courteously provided by several users of AMDC) and to simulated data. In all cases,

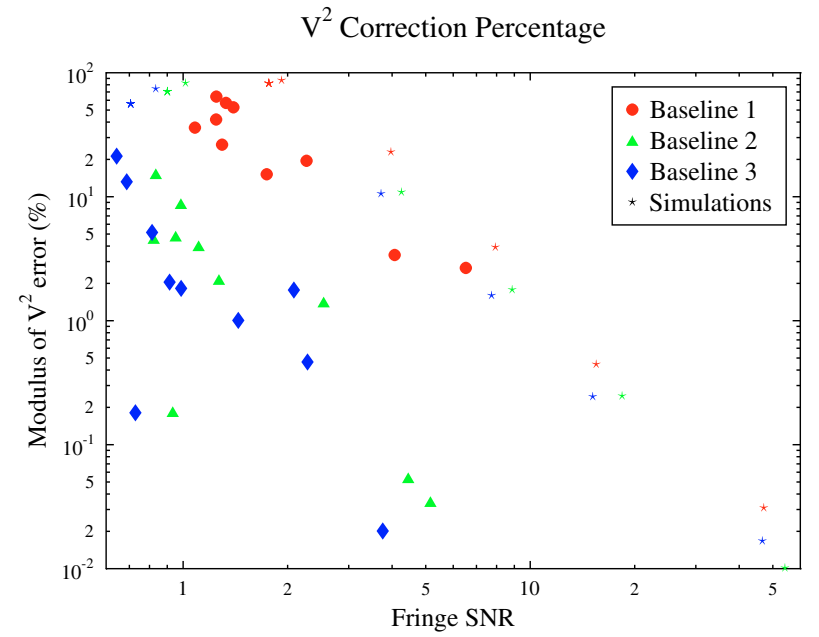

Fig. 9. Percentage correction of the instrumental squared visibility on different data sets and synthetic simulations. The artifact effect becomes dominant as detection limit is approached.

we have found similar improvements after fringing removal. In Fig. 9 we show a plot of the correction percentage of the instrumental visibilities, as a function of the fringe SNR. We note that the artifact effect tends to increase with decreasing fringe SNR.

We have also found that the locations of the artifact peaks in the frequency plane are very similar in all the observations we have analyzed; this fact gives an indication of the stability of the unknown sources of the correlated noise during the time period covered by the observations (from February 2005 to June 2006).

\section{Conclusions}

While analyzing VLTI-AMBER observations, a fringing artifact (correlated noise on the instrument detector) has been found on the data frames; this biases the visibility computation for low SNR targets. This spurious pattern produces a series of spatial frequency peaks in the power spectrum of the frames, some of which fall at the same baseline frequencies of the interferometric signal, thus altering the computed visibilities. We have shown that this effect becomes more important in faint sources, causing the instrumental visibility to be overestimated when dealing with targets near magnitude limit. We have presented a procedure to restore the unbiased data, and we provide to the community the algorithm of this method implemented in a software program called AMDC (freely available at http://www .mporzio.astro.it/ licausi/AMDC/), so that AMBER users can perform optimal data reduction for observations obtained in poor instrumental conditions. AMDC works as a pre-processing tool to be run on the original AMBER frames before the standard amdlib extraction is performed. The software is planned to be implemented in a future version of amdlib. 
Acknowledgements. The authors are very grateful to M. Benisty, F. Cusano, A. Domiciano de Souza, G. Duvert, O. Hernandez, A. Isella, F. Malbet and W. de Wit for providing their observation data sets, which have been joined to our data for completing the diagram in Fig. 9. We also thank G. Duvert for providing very useful numerical simulations that we have used to test our algorithm and U. Beckmann for explanations of the AMBER readout.

\section{References}

Altariba, E., Duvert, G., \& Zins, G. 2006, AMBER data reduction software design description document - VLT-TRE-AMB-15830-4107

Beckmann, U. 2007, Report of the AMBER detector intervention from September 10 to 19,2007
Gai, M., Menardi, S., Cesare, S., et al. 2004, in New Frontiers in Stellar Interferometry, ed. A. Wesley, Traub. Bellingham, WA: The International Society for Optical Engineering, Proc. Spie, 5491, 528

Koresko, C. D., Beckwith, S. V. W., Ghez, A. M., Matthews, K., \& Neugebauer, G. 1991, AJ, 102, 2073

Mardones, P. 2007, AMBER image interferences report

Millour, F., Tatulli, E., Chelli, A. E., et al. 2004, in New Frontiers in Stellar Interferometry, ed. A. Wesley, Traub. Bellingham, WA: The International Society for Optical Engineering, Proc. Spie, 5491, 1222

Petrov, R. G., Malbet, F., Weigelt, G., et al. 2007, A\&A, 464, 1

Tatulli, E., Millour, F., Chelli, A., et al. 2007, A\&A, 464, 29 